\title{
Antenatal care during the pandemic in India: the problem and the solutions
}

\section{Keywords: antenatal care, pandemic situation, maternal mortality}

\section{Introduction}

COVID-19 pandemic has posed unprecedented challenges to public health, food systems and economic structure of the world leading to dramatic loss of human life and income. ${ }^{1}$ The obstacles were not only in treating patients infected by the virus but also for seeking medical care for other non-COVID pathological and physiological health conditions. Antenatal care is one such non-emergency yet essential health service which got overlooked and hence was compromised. During the pre-COVID times, antenatal care in India was either based on traditional care model (involving 12-14 visits) ${ }^{2}$ or WHO-2016 based recommendations (involving at least 8 visits). ${ }^{3}$ Such models were formulated to reduce perinatal mortality and improve women's experience of care during pregnancy. It assists in screening, diagnosing and managing the risk factors that might adversely affect the pregnant women and pregnancy outcome. ${ }^{4}$ However, in the current pandemic situation such frequent visits to hospital for seeking care; will expose the expectant mothers and their fetuses to COVID-19 infection. Pregnant women because of physiological alterations in immune and cardiorespiratory systems fall under vulnerable group to develop complications due to virus even though are not at an increased risk of getting infected as compared to the general population. ${ }^{5}$

\section{The problem}

According to the data released by UNICEF, India is projected to record the highest number of births since Covid-19 was declared a pandemic in March 2020, with 20.1 million babies expected to be born between March and December. ${ }^{6}$ This gives us a rough estimate that a large number of women are deprived of appropriate prenatal care during their pregnancy. The factors affecting these services are present on both supply and demand sides. As majority of health care delivery facilities are dedicated to COVID-19 care, staff getting engaged in emergency COVID-19 duties, several staff becoming positive and mandatory quarantines of staff have restricted delivery of ante-natal care. On the demand side, lack of information about availability of services, increased fears and concerns of being exposed, movement restrictions, loss of income, and social factors caused hindrance in seeking continuous antenatal care. ${ }^{7}$

There are many concerns for pregnant women from lower socio-economic status. Reorganizations of government hospitals for management of patients affected by the pandemic and closure of routine antenatal OPDs have prevented patients to approach hospitals. This has led to decline in number of ANC registrations to approximately 80 percent in April-May 2020 as compared to the corresponding period in $2019 .{ }^{8}$ Routine laboratories and sonography services are also adjourned further affecting the routine antenatal care. Each antenatal visit involves detailed physical and obstetric examination and hence, non-availability of such resources has led to delay in screening and diagnosis of preventable pregnancy related health problems like anaemia, gestational diabetes, gestational hypertension, pre-eclampsia, fetal growth restriction/death, breast problems, and any other heart, liver or kidney diseases. ${ }^{9}$ Number of
Volume 7 Issue I - 202 I

\author{
Radhika Aggarwal,' Arun Kr Sharma, ${ }^{2}$ Kiran \\ Guleria' \\ 'Department of Obstetrics \& Gynecology, University College of \\ Medical Sciences, India \\ ${ }^{2}$ Department of Community Medicine, University College of \\ Medical Sciences, India
}

Correspondence: Arun Kr Sharma, Department of

Community Medicine, University College of Medical Sciences,

Delhi, India, Email arsharma62@gmail.com

Received: January 04, 202I | Published: February 22, 202 I

emergency admissions for pregnancy related complications like raised BP, seizures, decreased/absent fetal movements, deranged blood sugars, bleeding PV, meconium staining of liquor, oligohydroamnios, fetal distress, IUD and still birth has increased as compared to preCOVID era. ${ }^{10}$ Many patients also present with severe anaemia and shock as patients did not have a regular supply of IFA and calcium. There has been 2.5-fold rise in admission to intensive care unit of pregnant women during the pandemic. ${ }^{11}$ According to a model studied on Asia-pacific region which includes India, in the best-case scenario, due to $20 \%$ decline in use of sexual and reproductive health services: births assisted by skilled health-care providers, including midwives; births taking place in health facilities; and access to contraception, there will be $17 \%$ increase in maternal mortality ratio. In the worstcase scenario with $50 \%$ decline in use of services, there will be $43 \%$ increase in maternal mortality. ${ }^{12}$ Delay in obstetric ultrasound has led to challenges in early diagnosis, dating of pregnancy and ensuring fetal well-being. ${ }^{9}$ Screening of genetic disorders has also become difficult as it is considered non-essential as per the changed pandemic guidelines of antenatal care by FOGSI. ${ }^{13}$ However, it may have serious impact on outcome of pregnancies previously affected by any genetic or metabolic disorder. Invasive fetal diagnostic and therapeutic procedures are also put on hold. Health education for nutrition, exercise, birth preparedness, breast feeding and contraception and alleviating pregnancy related anxiety has also become difficult.

Not only the physical health, but mental health has been equally impacted. There has been a sharp rise in obstetric abuse and violence at institutions and home hampering the maternal mental health. ${ }^{14}$ Social ignorance, lack of family support and increased household workload has increased mental pressure and has disturbed lifestyle. There has also been difficulty in availing obstetric services during labour. Most of the ambulance services are diverted for COVID-19 related activities and with suspension of transportation facilities, women in labor fond it difficult to reach a healthcare facility. There have been reported cases of women giving birth in ambulances because of delay in transport. ${ }^{15}$ Even if they reach a facility on time, they have to get COVID-19 testing done and in case they report positive, they are referred to a 
COVID-19 designated facility for delivery which led to further delay. Number of home deliveries has also increased as indicated by decrease in number of institutional deliveries to approximately 78 percent in April-May 2020 corresponding to the figures in AprilMay 2019. ${ }^{8}$ Also, the number of childbirths registered in hospitals in both public and private facilities across India stood at 17,17,500 in March last year while this year the number dropped to 9,71,782. ${ }^{8}$

Women from higher income families are anxious because of cancellation of their routine appointments, fear of increased risk of COVID-19 infection at hospital and lack of proper infrastructure and trained staff because of lower physical attendance of private practitioners in their clinics and private hospitals. ${ }^{15}$ There has been significant change in hospital landscape in terms of consultations, visits and treatment. The number of visits has been cut down, duration of contact reduced and only a single attendant is allowed to come along. Patients come with prior appointment because of which there is delay in seeking antenatal care. Tele consultations have been set up for some visits but no fixed model has come into place. If a woman come out to be COVID-19 positive, she is then referred to a COVID designated facility.

\section{The possible solutions}

Since large numbers of patients are not able to avail routine antenatal services, they have suffered in terms of pregnancy care and outcome. Hence, there is immediate need for strict regulations to promote continuous and optimum antenatal care. Some measures that can be adopted to improve the care provided are- promoting COVID-19 related measures to prevent the transmission of infection, improving transportation facility for ANC visits and that of patients in labor, by designated ambulances for such need and by increasing the number of facilities for women for antenatal checkups. Each ANC visit can be clubbed with sonography and blood tests that need to be carried out for that woman in order to reduce hospital visits. Women can also be taught to monitor vitals and maintain a DFMC chart at home. Intelligent and smart use of technology in the form of teleconsultations for each missed ANC visit to prevent missing out on important signs and symptoms that may require hospital admission. Telephonic and online help groups can also guide women during their pregnancy as to where is the nearest facility, the services available, transport mediums and mental and social support needed. ${ }^{9}$ Mobile applications can be developed which notify women about the dates of their each antenatal visit, where blood pressure, blood sugar, and DFMC can be charted, routine and obstetric investigations can be recorded, help can be availed in form of videos on home monitoring, danger signs during pregnancy and emergency contact number and location of nearest health facility. Women can be made aware of such facilities by written/pre-recorded voice messages on telephones, social media, newspaper, radio and advertisements. Small teams of Accredited Social Health Activists (ASHA) and anganwadi workers can be made that can reinforce these messages to small communities in rural areas as they are their trustworthy companions by maintaining appropriate COVID-19 related precautions. ANM and other health workers can also be trained in the workings of teleconsultations and usage of such applications. Women can approach these workers by telephone/online services who can connect them to a specialist when needed. They can also be trained to identify danger signs and refer women as and when required. ANM can also direct a COVID-19 infected pregnant female to a health facility by arranging ambulance services and providing social support.

There is a need for collaboration of various authorities at regional, state and national level in order to promote and provide care needed by pregnant women. mHealth (mobile health) initiatives need to be integrated into national health systems. ${ }^{16}$ Political responsibility is to create infrastructure and regulatory frameworks to provide such services. It will support confidentiality and will shape finance models. ${ }^{16,17}$ There should also be clear laid out roles at each level to maintain smooth functioning. The development of generic public platforms can provide the processing power, storage, security, access control and other services. ${ }^{16,18}$ There can be challenges in delivery of such antenatal care such as concerns about reliability of providing information online or on telephones, patient and provider satisfaction, gaps in contact and perceived liability. ${ }^{19,20}$ These can be combated by improving the quality of these online platforms. Women too need to be vigilant during their pregnancy and co-operate to receive optimum antenatal care. The fight is long and for unpredictable timeline, hence such measures needs to be made 'new normal' practices that can be implemented in post-COVID era too.

\section{Acknowledgments}

None.

\section{Conflicts of interest}

Author declares that there is no conflict of interest.

\section{Funding}

None.

\section{References}

1. FAO. Impact of COVID-19 on people's livelihoods, their health and our food systems. 2021

2. Park K. Preventive medicine in obstetrics, paediatrics and geriatrics. In: Park K, ed. Park's Textbook of Preventive and Social Medicine, 23rd ed. New Delhi: Bhanot Publishers; 2015. 523 p.

3. WHO. Recommendation on antenatal care contact schedules

4. Sylvia Patience Ngxongo T. Basic antenatal care approach to antenatal care service provision. Selected Topics in Midwifery Care. 2019.

5. Avila W, Carvalho R. COVID-19: A New Challenge in Pregnancy and Heart Disease. Arquivos Brasileiros de Cardiologia. 2020;115(1):1-4.

6. UNICEF. Pregnant Mothers and Babies Born During COVID-19 Pandemic Threatened By Strained Health Systems and Disruptions in Services. 2020.

7. Motihar R. The impact of COVID-19 on reproductive health services. India Development Review. 2020.

8. Health Management Information Systems (HMIS) - MEASURE Evaluation. 2020.

9. Zangmo R, Kumari A, Garg D, et al. Redesigning routine antenatal care in low resource setting during COVID-19 pandemic. J Family Med Prim Care. 2020;9(9):4547-4551.

10. Vasudhar A, Anita S, Patil GL, et al. Obstetric emergencies during the COVID-19 lockdown period: a case series. Int J Reprod Contracept Obstet Gynecol. 2020;9(11):4682-4687.

11. Goyal M, Singh P, Singh K, et al. The effect of the COVID-19 pandemic on maternal health due to delay in seeking health care: Experience from a tertiary center. Int J Gynaecol Obstet. 2020.

12. World Economic Forum. Coronavirus is leaving pregnant women with tough choices and bleaker outcomes. 2021.

13. Chawla D, Chirla D, Dalwai S, et al. Perinatal-Neonatal Management of COVID-19 Infection - Guidelines of the Federation of Obstetric and Gynaecological Societies of India (FOGSI), National Neonatology Forum of India (NNF), and Indian Academy of Pediatrics (IAP). Indian Pediatr. 2020;57(6):536-548. 
14. The Wire. Obstetric Violence During COVID-19 Is yet Another Challenge for Indian Women. 2021.

15. The Wire. COVID-19 Lockdown: Guidelines Are Not Enough to Ensure Pregnant Women Receive Care. 2020.

16. Barkman C, Weinehall L. Policymakers and Health: roles and expectations, with observations from Ethiopia, Ghana and Sweden. Global Health Action. 2017;10(sup3):1337356..

17. World Health Organization and International Telecommunication Union National eHealth strategy toolkit. Geneva: WHO; 2012.
18. University of Cambridge Mobile communications for medical care. A study of current and future healthcare and health promotion applications, and their use in China and elsewhere. Cambridge (UK); 2020.

19. Dowswell T, Carroli G, Duley L, et al. Alternative versus standard packages of antenatal care for low-risk pregnancy. Cochrane Database Syst Rev. 2015;2015(7): CD000934.

20. Carroli G, Villar J, Piaggio G, et al. WHO Antenatal Care Trial Research Group. WHO systematic review of randomised controlled trials of routine antenatal care. Lancet. 2001;357(9268):1565-1570. 